\title{
Global trends of antimicrobial susceptibility to ceftaroline and ceftazidime-avibactam: a surveillance study from the ATLAS program (2012-2016)
}

Hui Zhang ${ }^{\dagger}$, Yingchun $\mathrm{Xu}^{\dagger}$, Peiyao Jia ${ }^{\dagger}$, Ying Zhu, Ge Zhang, Jingjia Zhang, Simeng Duan, Wei Kang, Tong Wang, Ran Jing, Jingwei Cheng, Yali Liu and Qiwen Yang*

\begin{abstract}
Background: This study reports the global trends of antimicrobial susceptibility to ceftaroline and ceftazidime-avibactam using data from the Antimicrobial Testing Leadership and Surveillance (ATLAS) program between 2012 and 2016.

Methods: For the 2012-2016 ATLAS program, 205 medical centers located in Africa-Middle East $(n=12)$, Asia-Pacific $(n=32)$, Europe $(n=94)$, Latin America $(n=26)$, North America $(n=31)$, and Oceania $(n=10)$ consecutively collected the clinical isolates. The minimum inhibitory concentrations (MICs) and in vitro susceptibilities to ceftaroline and ceftazidime-avibactam were assessed using the Clinical and Laboratory Standards Institute (CLSI) 2019and European Committee on Antimicrobial Susceptibility Testing (EUCAST) 2019 guidelines.

Results: Between 2012 and 2016, 176,345 isolates were collected from around the globe and included in the analysis. Regarding Gram-negative bacteria, ceftazidime-avibactam demonstrated high susceptibility (>90\%) against Enterobacteriaceae and Pseudomonas aeruginosa, with increased antimicrobial activity observed from the addition of avibactam (4 mg/L) to ceftazidime. Regarding Gram-positive bacteria, ceftaroline showed $>90 \%$ susceptibility against Staphylococcus aureus, Streptococcus pneumoniae, a-and $\beta$-hemolytic Streptococcus. The antimicrobial susceptibilities to ceftaroline and ceftazidime-avibactam were mostly stable from 2012 to 2016, but the susceptibilities to ceftazidime-avibactam to carbapenem-resistant (CR) Klebsiella pneumonia (88.4-81.6\%) and to CR-P. aeruginosa (89.6-72.7\%) decreased over time. In terms of regional difference, the susceptibilities of methicillin-resistant S. aureus to ceftaroline in Asia and of CR-K. pneumonia to ceftazidime-avibactam in Asia/Africa-Middle East were lower compared with other regions, while the susceptibility of CR-P. aeruginosa to ceftazidime-avibactam in North America was higher.
\end{abstract}

Conclusion: The addition of avibactam improves the activity of ceftazidime against Enterobacteriaceae and P. aeruginosa. The global antimicrobial susceptibilities to ceftaroline and ceftazidime-avibactam were, in general, stable from 2012 to 2016, but a marked reduction in the susceptibilities of specific species and CR-P. aeruginosa to ceftazidimeavibactam was observed.

*Correspondence: yangqiwen@pumch.cn

${ }^{\dagger}$ Hui Zhang, Yingchun Xu and Peiyao Jia have contributed equally to this

work

Department of Clinical Laboratory, Peking Union Medical College

Hospital, Peking Union Medical College, Chinese Academy of Medical

Science, Beijing 100730, China

(c) The Author(s) 2020. Open Access This article is licensed under a Creative Commons Attribution 4.0 International License, which permits use, sharing, adaptation, distribution and reproduction in any medium or format, as long as you give appropriate credit to the original author(s) and the source, provide a link to the Creative Commons licence, and indicate if changes were made. The images or other third party material in this article are included in the article's Creative Commons licence, unless indicated otherwise in a credit line to the material. If material is not included in the article's Creative Commons licence and your intended use is not permitted by statutory regulation or exceeds the permitted use, you will need to obtain permission directly from the copyright holder. To view a copy of this licence, visit http://creativecommons.org/licenses/by/4.0/. The Creative Commons Public Domain Dedication waiver (http://creativeco mmons.org/publicdomain/zero/1.0/) applies to the data made available in this article, unless otherwise stated in a credit line to the data. 
Keywords: Ceftaroline, Ceftazidime-avibactam, Antibiotics, Surveillance, Antimicrobial resistance

\section{Introduction}

The rapidly increasing and global spreading of the resistance of bacteria to antibiotics in recent years is a serious challenge for clinicians and a global health crisis [1]. Multi-drug resistance in both Gram-negative and -positive bacteria often leads to untreatable infections using conventional antibiotics, and even last-resort antibiotics are losing their power [2]. The increases in the occurrence of infections caused by third-generation cephalosporin- and carbapenem-resistant (CR)-Enterobacteriaceae, CR-Pseudomonas aeruginosa, and CRAcinetobacter baumannii are of particular concern since they are associated with tremendously increased mortality and morbidityrates $[3,4]$. Recently, the World Health Organization has rated CR-Enterobacteriaceae, CR-P. aeruginosa, and CR-A. baumannii as top critical-priority resistant bacteria, outweighing methicillin-resistant Staphylococcus aureus [5]. Consequently, updated epidemiological data on antibiotic resistance is needed to adapt the treatment strategies to the reality, which changes at an alarming rate [4, 6-8].

Ceftaroline is a fifth-generation broad-spectrum cephalosporin. It is mainly active against methicillin-resistant S. aureus and Gram-positive bacteria, but also against Gram-negative bacteria [9]. Ceftarolineis indicated for community-acquired pneumonia and complicated skin infections [10-13]. Avibactam is a diazabicyclooctane derivative antibiotic that can reversibly inhibit $\beta$-lactamase enzymes, including Ambler class A (ESBL and $\mathrm{KPC}$ ), class $\mathrm{C}$, and partial class D (including OXA-1, OXA-10, and OXA-48-like) enzymes by covalent acylation of the active-site serine residue [14]. Ceftazidimeavibactam is a novel $\beta$-lactam $/ \beta$-lactamase inhibitor combination that has shown potency against a wide variety of CR-Enterobacteriaceae. Ceftazidime-avibactam has been approved for the management of complicated urinary tract infections, complicated intra-abdominal infections, hospital-acquired pneumonia, and infections from aerobic Gram-negative bacteria with limited treatment options [15].

Ceftaroline and ceftazidime-avibactam are relatively novel antibiotics that show promises in the control of antibiotic-resistant pathogens. They are readily available around the globe. The patterns of resistance to ceftaroline and ceftazidime-avibactam around the globe remain to be defined exactly and represent crucial data for monitoring global health threats. Therefore, this study aimed to: (1) examine the in vitro activities of ceftaroline, ceftazidime-avibactam, and various comparative agents from 2012 to 2016 using the data from a global antibiotic surveillance program, the Antimicrobial Testing Leadership And Surveillance (ATLAS) program; and (2) compare the susceptibility profile of various pathogen species over time and across different regions of the world, with an emphasis on antibiotic-resistant pathogens.

\section{Materials and methods \\ Bacterial isolates}

For the 2012-2016 ATLAS program, 205 medical centers located in Africa-Middle East $(n=12)$, Asia-Pacific $(\mathrm{n}=32)$, Europe $(\mathrm{n}=94)$, Latin America $(\mathrm{n}=26)$, North America $(n=31)$, and Oceania $(n=10)$ contributed to the consecutive collection of clinical isolates. The specimens were obtained from inpatients with specific types of infections (skin and skin structure infection, intraabdominal infection, urinary tract infection, lower respiratory tract infection, and blood infection). The pathogens were isolated and identified by each participating center, stored in tryptic soy broth with glycerol at $-70{ }^{\circ} \mathrm{C}$, and shipped to International Health Management Associates, Inc. (IHMA; Schaumburg, IL, USA) for susceptibility testing. The present study only included the isolates considered to be the potential pathogen of the patient's infection. If multiple samples were taken from the same patient during an infectious event, only the first positive sample for this infectious event was included in the ATLAS program. The pathogen identification was confirmed by MALDI-TOF at IHMA (Schaumburg, IL, USA) prior to susceptibility testing. Methicillin-resistant $S$. aureus is defined in this study as $S$. aureus resistant to oxacillin.

\section{Antimicrobial susceptibility testing}

IHMA (Schaumburg, IL, USA) carried out all antimicrobial susceptibility tests using the broth microdilution method. The minimum inhibitory concentrations (MICs) were interpreted using the Clinical and Laboratory Standards Institute (CLSI) 2019 and the European Committee on Antimicrobial Susceptibility Testing (EUCAST) 2019 breakpoints [16, 17]. Tigecycline was interpreted using the Food and Drug Administration and EUCAST 2019 interpretative breakpoints. Ceftaroline, ceftazidime-avibactam (avibactam at a fixed concentration of $4 \mathrm{mg} / \mathrm{L}$ ), and the following comparator agents were tested:ceftazidime, cefepime, penicillin, ampicillin, piperacillin-tazobactam, doripenem, imipenem, meropenem, levofloxacin, moxifloxacin, clindamycin, erythromycin, vancomycin, teicoplanin, linezolid, daptomycin, 
gentamicin, tigecycline, minocycline, trimethoprim-sulfamethoxazole, amikacin, colistin, aztreonam, quinupristin-dalfopristin, andoxacillin. In the present study, the data were analyzed for Escherichia coli, Klebsiella pneumoniae, Enterobactercloacae, Citrobacter freundii, Proteus mirabilis, $P$. aeruginosa, A. baumannii, $S$. aureus, Streptococcus pneumoniae, $\alpha$ - and $\beta$-hemolytic Streptococcus, coagulase-negative Staphylococcus, Enterococcus faecalis, and Enterococcus faecium, as well as resistant species including CR-E. coli, CR-K. pneumoniae, CR-Enterobacter cloacae, CR-P. aeruginosa, CR-A. baumannii, methicillin-resistant $S$. aureus, and penicillin-resistant S. pneumoniae. All tests included quality control strains from the American Type Culture Collection (ATCC; Manassas, VA, USA). Escherichia coli ATCC 25922, K. pneumoniae ATCC 700603, P. aeruginosa ATCC 27853, S. aureus ATCC 29213, and S. pneumoniae ATCC 49619 were used for quality control according to the CLSI 2019 guidelines. All quality control results were within the published ranges.

\section{Results \\ Sample retrieval}

A total of 176,345 isolates were collected between 2012 and 2016. The numbers of isolates of each species group tested are listed in Tables 1 and 2. The largest number of isolates were collected from patients $>60$ years $(82,518$, $46.8 \%)$ and $31-60$ years $(59,428,33.7 \%)$, followed by patients < 18 years $(19,446,11.0 \%)$ and $19-30$ years $(13,350,7.6 \%)$. Regarding the infection types, 64,032 (36.3\%) isolates were collected from skin and skin structure infections, 52,077 (29.5\%) from lower respiratory tract infections, 26,868 (15.2\%) from urinary tract infections, 12,847 (7.3\%) from intra-abdominal infections, and 11,930 (6.8\%) from the blood. In regard to hospital location, 74,554 (42.3\%), 32,430 (18.4\%), 17,024 (9.7\%), $16,339(9.3 \%), 10,130(5.7 \%)$, and $8200(4.6 \%)$ isolates were from patients in the general medical wards, general surgical wards, emergency rooms, medical intensive care unit (ICUs), surgical ICUs, and general pediatric wards, respectively.

\section{In vitro activities of ceftaroline and ceftazidime-avibactam} against Gram-negative bacteria from 2012 to 2016

Table 1 (Gram-negative) and 2 (Gram-positive) show the in vitro activities of ceftaroline, ceftazidime-avibactam, and comparators against the selected bacteria. Ceftazidime-avibactam demonstrated high activities against all tested Gram-negative bacteria (CLSI/EUCAST 2019 susceptibility, 91.9-99.8\%). The susceptibility of $A$. baumannii was not calculated because of the absence of a breakpoint, but the MICs of this antibiotic were higher for $A$. baumannii than for the other bacteria $\left(\mathrm{MIC}_{50} /\right.$
$\mathrm{MIC}_{90}, 32 / 128 \mathrm{mg} / \mathrm{L}$ ). The addition of avibactam drastically increased the activity of ceftazidime against $E$. coli, $K$. pneumoniae, E. cloacae, $C$. freundii, and $P$. aeruginosa (CLSI 2019 susceptibilities to ceftazidime alone, 64.379.2\%) whereas a trend of decreased MIC was observed for A. baumannii, as indicated by a twofold reduction in $\mathrm{MIC}_{90}$ (ceftazidime, $\mathrm{MIC}_{50} / \mathrm{MIC}_{90}, 64 / 256 \mathrm{mg} / \mathrm{L}$ ). Regarding the comparator agents, the susceptibility of Enterobacteriaceae was, in general, high for carbapenems and tigecycline (>90\%). For A. baumannii, the most potent antibiotics were colistin and tigecycline $\left(\mathrm{MIC}_{50} /\right.$ $\mathrm{MIC}_{90}, 1 / 2 \mathrm{mg} / \mathrm{L}$ ), with $\mathrm{aMIC}_{50}$ of $\geq 8$ and $\mathrm{aMIC}_{90}$ of $\geq 16 \mathrm{mg} / \mathrm{L}$ observed for all other tested agents.

Regarding resistant Gram-negative strains, the activities of ceftazidime-avibactam were moderate for CR-E. coli $\left(\mathrm{MIC}_{50} / \mathrm{MIC}_{90}, 0.5 / 256 \mathrm{mg} / \mathrm{L}\right)$, CR-K. pneumoniae $\left(\mathrm{MIC}_{50} / \mathrm{MIC}_{90}, 1 / 256 \mathrm{mg} / \mathrm{L}\right.$ ), and CR-P. aeruginosa $\left(\mathrm{MIC}_{50} / \mathrm{MIC}_{90}, 4 / 64 \mathrm{mg} / \mathrm{L}\right)$ and low for CR-E. cloacae and CR-A. baumannii $\left(\mathrm{MIC}_{50} / \mathrm{MIC}_{90}, 64-128 / 256 \mathrm{mg} / \mathrm{L}\right.$ ) (Table 3). Regarding the comparator agents, the susceptibilities of CR-E. coli, CR-K. pneumoniae, CR-E. cloacae, CR-P. aeruginosa, and CR-A. baumannii were low for the vast majority of the tested antibiotics. Good potency was observed for tigecycline against all tested Enterobacteriaceae $\left(\mathrm{MIC}_{50} / \mathrm{MIC}_{90}, 0.25-1 / 1-4 \mathrm{mg} / \mathrm{L}\right)$, and for colistin against CR-E. coli, CR-E. cloacae, CR-P. aeruginosa, and CR-A. baumannii $\left(\mathrm{MIC}_{50} / \mathrm{MIC}_{90}, 0.5-1 / 1-2 \mathrm{mg} / \mathrm{L}\right.$ ).

The susceptibilities to the various antibiotics against Gram-negative bacteria (total, regardless of drug resistance) were in general comparable using CLSI 2019 and EUCAST 2019 breakpoints, except for imipenem and tigecycline against $P$. mirabilis (Table 1). Nevertheless, the susceptibilities of many resistant species were lower using the EUCAST 2019 breakpoints compared with the CLSI 2019 breakpoints. For example, the susceptibilities of CR-E. coli (72.3\% vs. $40.5 \%)$ and CR-E. cloacae $(42.3 \%$ vs. $21.9 \%)$ to ceftazidime-avibactam, and the susceptibilities of CR-E. coli, CR-K. pneumoniae, CR-E. cloacae, and CR-P. aeruginosa to levofloxacin, tigecycline, and amikacin (all with a $>10 \%$ difference) were noticeably lower when the EUCAST 2019 breakpoints were applied (Table 3).

\section{In vitro activities of ceftaroline and ceftazidime-avibactam} against Gram-positive bacteria from 2012 to 2016

In the Gram-positive strains, ceftaroline showed more than $90 \%$ susceptibility rates of $S$. aureus, S. pneumoniae, $\alpha$-hemolytic Streptococcus, and $\beta$-hemolytic Streptococcus (CLSI 2019). TheMIC ${ }_{50} / \mathrm{MIC}_{90}$ of ceftaroline for coagulase-negative Staphylococcus and E. faecalis were $0.25 / 1 \mathrm{mg} / \mathrm{L}$ and $1 / 16 \mathrm{mg} / \mathrm{L}$, respectively. Ceftaroline demonstrated low activity against E. faecium $\left(\mathrm{MIC}_{50} / \mathrm{MIC}_{90}, 64 / 64 \mathrm{mg} / \mathrm{L}\right.$ ) (Table 2). 
Table 1 In vitro susceptibilities of Gram-negative strains obtained from the ATLAS program, 2012-2016

\begin{tabular}{|c|c|c|c|c|c|c|c|c|c|c|}
\hline \multirow[t]{2}{*}{ Organism/antibiotic } & \multirow[t]{2}{*}{ No. of isolates } & \multirow[t]{2}{*}{$\mathrm{MIC}_{50}$} & \multirow[t]{2}{*}{$\mathrm{MIC}_{90}$} & \multirow[t]{2}{*}{ MIC range } & \multicolumn{3}{|c|}{$\mathrm{CLSI}^{\mathrm{a}}$} & \multicolumn{3}{|c|}{ EUCAST } \\
\hline & & & & & $\mathrm{S} \%$ & $1 \%$ & $\mathrm{R} \%$ & $\mathrm{~S} \%$ & $1 \%$ & $\mathrm{R} \%$ \\
\hline \multicolumn{11}{|l|}{ Escherichia coli } \\
\hline Ceftaroline & 21903 & 0.12 & 256 & $0.015-256$ & 66.5 & 2.6 & 30.9 & 66.5 & 0 & 33.5 \\
\hline Ceftazidime-avibactam & 21903 & 0.12 & 0.25 & $0.015-256$ & 99.8 & 0 & 0.2 & 99.8 & 0 & 0.2 \\
\hline Ceftazidime & 21903 & 0.25 & 32 & $0.015-256$ & 79.2 & 3.0 & 17.8 & 74.0 & 5.1 & 20.8 \\
\hline Cefepime & 21903 & 0.12 & 32 & $0.12-32$ & 76.2 & 4.7 & 19.1 & 74.6 & 3.6 & 21.8 \\
\hline Pip-taz & 21903 & 2 & 16 & $0.25-256$ & 90.3 & 4.6 & 5.1 & 84.8 & 5.4 & 9.8 \\
\hline Doripenem & 21903 & 0.03 & 0.06 & $0.008-16$ & 99.6 & 0.1 & 0.3 & 99.6 & 0.1 & 0.3 \\
\hline Imipenem & 21903 & 0.25 & 0.25 & $0.03-16$ & 99.1 & 0.4 & 0.5 & 99.5 & 0.4 & 0.2 \\
\hline Meropenem & 21903 & 0.03 & 0.06 & $0.004-16$ & 99.5 & 0.1 & 0.4 & 99.6 & 0.2 & 0.2 \\
\hline Levofloxacin & 21903 & 0.25 & 16 & $0.004-16$ & 62.3 & 1.7 & 36.0 & 58.8 & 2.8 & 38.4 \\
\hline Tigecycline & 21903 & 0.25 & 0.5 & $0.015-16$ & 99.8 & 0.2 & 0 & 99.0 & 0.8 & 0.2 \\
\hline Amikacin & 21903 & 2 & 8 & $0.25-64$ & 98.2 & 0.9 & 0.9 & 94.5 & 3.7 & 1.8 \\
\hline Colistin & 13964 & 0.5 & 1 & $0.06-16$ & NA & NA & NA & 99.5 & 0 & 0.5 \\
\hline Aztreonam & 21903 & 0.12 & 64 & $0.015-256$ & 76.0 & 3.1 & 20.9 & 72.3 & 3.7 & 24.0 \\
\hline \multicolumn{11}{|l|}{ Klebsiella pneumoniae } \\
\hline Ceftaroline & 18114 & 0.25 & 256 & $0.015-256$ & 57.5 & 2.0 & 40.5 & 57.5 & 0 & 42.5 \\
\hline Ceftazidime-avibactam & 18114 & 0.12 & 1 & $0.015-256$ & 98.8 & 0 & 1.2 & 98.8 & 0 & 1.2 \\
\hline Ceftazidime & 18114 & 0.25 & 128 & $0.015-256$ & 64.3 & 1.9 & 33.8 & 61.6 & 2.7 & 35.7 \\
\hline Cefepime & 18114 & 0.12 & 32 & $0.12-32$ & 65.1 & 6.0 & 28.9 & 63.7 & 3.2 & 33.1 \\
\hline Pip/taz & 18114 & 4 & 256 & $0.25-256$ & 73.0 & 7.8 & 19.2 & 64.4 & 8.6 & 27.0 \\
\hline Doripenem & 18114 & 0.06 & 0.5 & $0.008-16$ & 91.6 & 1.0 & 7.4 & 91.6 & 1.0 & 7.4 \\
\hline Imipenem & 18114 & 0.25 & 1 & $0.03-16$ & 90.3 & 1.9 & 7.8 & 92.2 & 2.4 & 5.5 \\
\hline Meropenem & 18114 & 0.06 & 0.5 & $0.004-16$ & 91.1 & 1.1 & 7.9 & 92.1 & 2.0 & 5.9 \\
\hline Levofloxacin & 18114 & 0.12 & 8 & $0.004-16$ & 73.2 & 3.1 & 23.7 & 61.8 & 9.1 & 29.1 \\
\hline Tigecycline & 18114 & 0.5 & 2 & $0.015-16$ & 96.4 & 3.1 & 0.5 & 88.2 & 8.2 & 3.6 \\
\hline Amikacin & 18114 & 1 & 8 & $0.25-64$ & 93.6 & 3.0 & 3.4 & 91.0 & 2.6 & 6.4 \\
\hline Colistin & 12884 & 0.5 & 1 & $0.06-16$ & NA & NA & NA & 96.3 & 0 & 3.7 \\
\hline Aztreonam & 18114 & 0.12 & 256 & $0.015-256$ & 64.2 & 1.0 & 34.8 & 62.4 & 1.8 & 35.8 \\
\hline \multicolumn{11}{|l|}{ Enterobacter cloacae } \\
\hline Ceftaroline & 4330 & 0.5 & 256 & $0.015-256$ & 60.0 & 3.1 & 37.0 & 60.0 & 0.0 & 40.1 \\
\hline Ceftazidime-avibactam & 4330 & 0.25 & 1 & $0.015-256$ & 97.8 & 0.0 & 2.2 & 97.8 & 0.0 & 2.2 \\
\hline Ceftazidime & 4330 & 0.5 & 128 & $0.015-256$ & 67.3 & 1.4 & 31.3 & 64.0 & 3.3 & 32.7 \\
\hline Cefepime & 4330 & 0.12 & 32 & $0.12-32$ & 78.5 & 8.8 & 12.7 & 73.3 & 10.8 & 15.9 \\
\hline Pip-taz & 4330 & 4 & 256 & $0.25-256$ & 75.3 & 8.0 & 16.7 & 69.9 & 5.4 & 24.7 \\
\hline Doripenem & 4330 & 0.06 & 0.25 & $0.008-16$ & 96.8 & 0.4 & 2.9 & 96.8 & 0.4 & 2.9 \\
\hline Imipenem & 4330 & 0.5 & 1 & $0.03-16$ & 93.1 & 3.5 & 3.4 & 96.6 & 2.0 & 1.5 \\
\hline Meropenem & 4330 & 0.06 & 0.12 & $0.004-16$ & 96.8 & 0.6 & 2.6 & 97.4 & 1.3 & 1.3 \\
\hline Levofloxacin & 4330 & 0.06 & 4 & $0.004-16$ & 88.8 & 2.8 & 8.4 & 80.8 & 5.7 & 13.5 \\
\hline Tigecycline & 4330 & 0.5 & 1 & $0.015-16$ & 96.3 & 3.2 & 0.5 & 90.1 & 6.2 & 3.7 \\
\hline Amikacin & 4330 & 2 & 4 & $0.25-64$ & 97.6 & 0.9 & 1.6 & 96.0 & 1.6 & 2.4 \\
\hline Colistin & 2889 & 0.5 & 1 & $0.12-16$ & NA & NA & NA & 93.7 & 0.0 & 6.3 \\
\hline Aztreonam & 4330 & 0.12 & 64 & $0.015-256$ & 68.2 & 1.4 & 30.4 & 65.8 & 2.4 & 31.8 \\
\hline \multicolumn{11}{|l|}{ Citrobacter freundii } \\
\hline Ceftaroline & 2327 & 0.25 & 128 & $0.015-256$ & 61.9 & 2.1 & 36.0 & 61.9 & 0 & 38.1 \\
\hline Ceftazidime-avibactam & 2327 & 0.12 & 0.5 & $0.015-256$ & 98.5 & 0 & 1.5 & 98.5 & 0 & 1.5 \\
\hline Ceftazidime & 2327 & 0.5 & 128 & $0.015-256$ & 68.0 & 1.9 & 30.1 & 64.3 & 3.8 & 32.0 \\
\hline Cefepime & 2327 & 0.12 & 4 & $0.12-32$ & 89.8 & 3.6 & 6.7 & 84.4 & 7.3 & 8.4 \\
\hline Pip-taz & 2327 & 4 & 128 & $0.25-256$ & 77.1 & 12.0 & 11.0 & 70.5 & 6.6 & 23.0 \\
\hline
\end{tabular}


Table 1 (continued)

\begin{tabular}{|c|c|c|c|c|c|c|c|c|c|c|}
\hline \multirow[t]{2}{*}{ Organism/antibiotic } & \multirow[t]{2}{*}{ No. of isolates } & \multirow[t]{2}{*}{$\mathrm{MIC}_{50}$} & \multirow[t]{2}{*}{$\mathrm{MIC}_{90}$} & \multirow[t]{2}{*}{ MIC range } & \multicolumn{3}{|c|}{$\mathrm{CLSI}^{\mathrm{a}}$} & \multicolumn{3}{|c|}{ EUCAST } \\
\hline & & & & & $\mathrm{S} \%$ & $1 \%$ & $\mathrm{R} \%$ & $\mathrm{~S} \%$ & $1 \%$ & $\mathrm{R} \%$ \\
\hline Doripenem & 2327 & 0.06 & 0.12 & $0.008-16$ & 97.9 & 0.3 & 1.8 & 97.9 & 0.3 & 1.8 \\
\hline Imipenem & 2327 & 0.5 & 2 & $0.03-16$ & 88.9 & 8.5 & 2.6 & 97.4 & 2.1 & 0.5 \\
\hline Meropenem & 2327 & 0.03 & 0.06 & $0.004-16$ & 97.7 & 0.5 & 1.8 & 98.2 & 1.2 & 0.6 \\
\hline Levofloxacin & 2327 & 0.12 & 4 & $0.008-16$ & 87.0 & 4.0 & 9.0 & 76.5 & 6.2 & 17.3 \\
\hline Tigecycline & 2327 & 0.5 & 1 & $0.015-8$ & 98.9 & 1.1 & 0 & 94.9 & 4.0 & 1.1 \\
\hline Amikacin & 2327 & 2 & 4 & $0.25-64$ & 98.4 & 0.4 & 1.2 & 97.1 & 1.3 & 1.6 \\
\hline Colistin & 1593 & 0.5 & 1 & $0.06-16$ & NA & NA & NA & 99.6 & 0 & 0.4 \\
\hline Aztreonam & 2327 & 0.25 & 64 & $0.015-256$ & 69.2 & 2.4 & 28.4 & 66.2 & 3.1 & 30.8 \\
\hline \multicolumn{11}{|l|}{ Proteus mirabilis } \\
\hline Ceftaroline & 3950 & 0.12 & 128 & $0.015-256$ & 79.4 & 2.0 & 18.6 & 79.4 & 0 & 20.6 \\
\hline Ceftazidime-avibactam & 3950 & 0.03 & 0.06 & $0.015-256$ & 99.7 & 0 & 0.3 & 99.7 & 0 & 0.3 \\
\hline Ceftazidime & 3950 & 0.06 & 1 & $0.015-256$ & 95.2 & 1.7 & 3.1 & 91.1 & 4.1 & 4.8 \\
\hline Cefepime & 3950 & 0.12 & 8 & $0.12-32$ & 88.2 & 3.4 & 8.5 & 86.9 & 2.9 & 10.3 \\
\hline Pip-taz & 3950 & 0.5 & 1 & $0.25-256$ & 98.5 & 0.9 & 0.6 & 97.7 & 0.8 & 1.5 \\
\hline Doripenem & 3950 & 0.25 & 0.5 & $0.008-16$ & 98.4 & 1.0 & 0.6 & 98.4 & 1.0 & 0.6 \\
\hline Imipenem & 3950 & 2 & 4 & $0.03-16$ & 25.8 & 45.9 & 28.3 & 71.7 & 27.7 & 0.6 \\
\hline Meropenem & 3950 & 0.06 & 0.12 & $0.004-16$ & 99.6 & 0.2 & 0.3 & 99.8 & 0.2 & 0.1 \\
\hline Levofloxacin & 3950 & 0.12 & 8 & $0.015-16$ & 76.6 & 5.5 & 17.9 & 64.9 & 4.5 & 30.6 \\
\hline Tigecycline & 3950 & 2 & 8 & $0.03-16$ & 52.2 & 37.3 & 10.5 & 20.9 & 31.3 & 47.8 \\
\hline Amikacin & 3950 & 4 & 8 & $0.25-64$ & 95.6 & 1.1 & 3.4 & 91.5 & 4.1 & 4.4 \\
\hline Colistin & 2412 & 16 & 16 & $0.25-16$ & NA & NA & NA & 0.5 & 0 & 99.5 \\
\hline Aztreonam & 3950 & 0.015 & 0.5 & $0.015-256$ & 95.9 & 0.8 & 3.3 & 93.1 & 2.9 & 4.1 \\
\hline \multicolumn{11}{|l|}{ Pseudomonas aeruginosa } \\
\hline Ceftaroline & 16014 & 16 & 256 & $0.015-256$ & NA & NA & NA & NA & NA & NA \\
\hline Ceftazidime-avibactam & 16014 & 2 & 8 & $0.015-256$ & 91.9 & 0 & 8.1 & 91.9 & 0 & 8.1 \\
\hline Ceftazidime & 16014 & 4 & 64 & $0.06-256$ & 76.7 & 4.6 & 18.8 & 76.7 & 0 & 23.4 \\
\hline Cefepime & 16014 & 4 & 32 & $0.12-32$ & 78.4 & 11.2 & 10.5 & 78.4 & 0 & 21.6 \\
\hline Pip-taz & 16014 & 8 & 256 & $0.25-256$ & 68.9 & 13.8 & 17.3 & 68.9 & 0 & 31.2 \\
\hline Doripenem & 16013 & 0.5 & 8 & $0.008-16$ & 74.3 & 7.6 & 18.2 & 67 & 7.2 & 25.8 \\
\hline Imipenem & 16014 & 2 & 16 & $0.03-16$ & 63.4 & 8.2 & 28.4 & 71.6 & 4.5 & 23.9 \\
\hline Meropenem & 16014 & 0.5 & 16 & $0.008-16$ & 72.5 & 6.0 & 21.5 & 72.5 & 11.9 & 15.6 \\
\hline Levofloxacin & 16014 & 1 & 8 & $0.004-16$ & 70.4 & 6.8 & 22.9 & 61.7 & 0 & 38.3 \\
\hline Amikacin & 16014 & 4 & 16 & $0.25-64$ & 90.4 & 2.7 & 6.9 & 85.9 & 4.5 & 9.6 \\
\hline Colistin & 12449 & 1 & 2 & $0.06-16$ & 96.6 & 0 & 3.4 & 96.6 & 0 & 3.4 \\
\hline Aztreonam & 16014 & 8 & 32 & $0.015-256$ & NA & NA & NA & 3.9 & 73.4 & 22.8 \\
\hline \multicolumn{11}{|l|}{ Acinetobacter baumannii } \\
\hline Ceftaroline & 3567 & 256 & 256 & $0.015-256$ & NA & NA & NA & NA & NA & NA \\
\hline Ceftazidime-avibactam & 3567 & 32 & 128 & $0.03-256$ & NA & NA & NA & NA & NA & NA \\
\hline Ceftazidime & 3567 & 64 & 256 & $0.015-256$ & 30.1 & 2.4 & 67.5 & NA & NA & NA \\
\hline Cefepime & 3567 & 32 & 32 & $0.12-32$ & 29.9 & 10.4 & 59.7 & NA & NA & NA \\
\hline Pip-taz & 3567 & 256 & 256 & $0.25-256$ & 25.4 & 3.7 & 70.9 & NA & NA & NA \\
\hline Doripenem & 3567 & 8 & 16 & $0.015-16$ & 33.2 & 1.4 & 65.4 & 30.4 & 2.8 & 66.8 \\
\hline Imipenem & 3567 & 16 & 16 & $0.03-16$ & 33.8 & 1.2 & 65.0 & 33.8 & 2.7 & 63.5 \\
\hline Meropenem & 3567 & 16 & 16 & $0.015-16$ & 32.8 & 1.6 & 65.6 & 32.8 & 3.5 & 63.7 \\
\hline Levofloxacin & 3567 & 8 & 16 & $0.03-16$ & 29 & 9.6 & 61.4 & 26.1 & 1.0 & 73 \\
\hline Tigecycline & 3567 & 1 & 2 & $0.015-16$ & NA & NA & NA & NA & NA & NA \\
\hline Amikacin & 3567 & 64 & 64 & $0.25-64$ & 42.5 & 5.8 & 51.7 & 40.2 & 2.3 & 57.5 \\
\hline Colistin & 2404 & 1 & 2 & $0.06-16$ & 94.3 & 0 & 5.7 & 94.3 & 0 & 5.7 \\
\hline
\end{tabular}


Table 1 (continued)

\begin{tabular}{|c|c|c|c|c|c|c|c|c|c|c|}
\hline \multirow[t]{2}{*}{ Organism/antibiotic } & \multirow[t]{2}{*}{ No. of isolates } & \multirow[t]{2}{*}{$\mathrm{MIC}_{50}$} & \multirow[t]{2}{*}{$\mathrm{MIC}_{90}$} & \multirow[t]{2}{*}{ MIC range } & \multicolumn{3}{|c|}{$\mathrm{CLSI}^{\mathrm{a}}$} & \multicolumn{3}{|c|}{ EUCAST } \\
\hline & & & & & $\mathrm{S} \%$ & $1 \%$ & $\mathrm{R} \%$ & $\mathrm{~S} \%$ & $1 \%$ & $\mathrm{R} \%$ \\
\hline Aztreonam & 3567 & 64 & 256 & $0.015-256$ & NA & NA & NA & NA & NA & NA \\
\hline
\end{tabular}

CLSI Clinical Laboratory and Standards Institute, EUCAST European Committee on Antimicrobial Susceptibility Testing, NA not applicable

a Cefepime CLSI susceptibility for Enterobacteriaceae adopted the susceptible, susceptible-dose-dependent, and resistant categories

Ceftazidime-avibactam showed low activity against coagulase-negative Staphylococcus, S. aureus, E. faecalis, and E. faecium $\left(\mathrm{MIC}_{50} / \mathrm{MIC}_{90}: 16-64 / 64 \mathrm{mg} / \mathrm{L}\right)$, moderate activity against $S$. pneumonia and $\alpha$-hemolytic Streptococcus $\left(\mathrm{MIC}_{50} / \mathrm{MIC}_{90}, 0.25 / 16 \mathrm{mg} / \mathrm{L}\right)$, and high activity against $\beta$-hemolytic Streptococcus $\left(\mathrm{MIC}_{50} /\right.$ $\left.\mathrm{MIC}_{90}, 0.025 / 0.5 \mathrm{mg} / \mathrm{L}\right)$. The addition of avibactam to ceftazidime was not associated with improved activities against the tested Gram-positive strains. For all tested Staphylococcus, Streptococcus, and Enterococcus, high susceptibility $(>90 \%)$ to linezolid, tigecycline, daptomycin, and vancomycin were observed (excepted for $E$. faecium to vancomycin). High activities (susceptibility, $>90 \%$ ) of levofloxacin and moxifloxacin were observed for Streptococcus.

Regarding the resistant Gram-positive strains, ceftaroline demonstrated high activities against methicillinresistant S. aureus (CLSI 2019susceptibility, 89.0\%) and penicillin-resistant S. pneumoniae (CLSI 2019susceptibility, 98.2\%), whereas ceftazidime-avibactam demonstrated limited activities $\left(\mathrm{MIC}_{50} / \mathrm{MIC}_{90}: 16-64 / 64 \mathrm{mg} / \mathrm{L}\right)$ (Table 3). For comparator agents, potent activity (CLSI 2019 susceptibility, >95\%) against methicillin-resistant $S$. aureus was observed for linezolid, tigecycline, vancomycin, teicoplanin, daptomycin, and trimethoprim sulfa, whereas the susceptibility of penicillin-resistant $S$. pneumonia (CLSI 2019 susceptibility, >95\%) was high to linezolid, tigecycline, vancomycin, levofloxacin, and moxifloxacin (Table 3).

The susceptibilities of Gram-positive bacteria (regardless of drug resistance) were similar between the CLSI 2019 and EUCAST 2019 breakpoints, except for the susceptibility of coagulase-negative Staphylococcus to teicoplanin and gentamicin. In terms of resistant strains, noticeably lower susceptibility of penicillin-resistant $S$. pneumoniae to ceftaroline (98.2\% vs. $86.8 \%)$ and meropenem (3.4\% vs. 100\%) was observed using ECUAST breakpoints as compared with CLSI 2019 breakpoints.

\section{Global trend of the susceptibilities of pathogens against ceftaroline and ceftazidime-avibactam from 2012 to 2016}

Figure 1 presents the trends of susceptibilities to ceftaroline against key bacterial species over time in different regions using the CLSI 2019 breakpoints. For E. coli (2012/2016:66.2\%/66.5\%), K. pneumoniae (2012/2016: 57.4\%/60.4\%), P. mirabilis (2012/2016: 78.7\%/81.2\%), S. aureus (2012/2016:92.5\%/95.1\%) and S. pneumonia (2012/2016:99.9\%/99.7\%), the overall global susceptibility to ceftaroline remained relatively stable in all regions from 2012 to 2016, but some decreases were observed in specific areas of the world. For E. coli, the susceptibilities were consistently higher in North America (77.1-82.0\%) and lower in Asia (45.1-53.0\%). Higher susceptibilities in North America were also observed for K. pneumoniae and P. mirabilis, and lower susceptibilities in Asia were observed for S. aureus. For E. cloacae, the global susceptibility gradually increased from $56.2 \%$ in 2012 to $64.6 \%$ in 2016. For $C$. freundii, the global susceptibility peaked at $69.1 \%$ in 2014 , decreased slightly in 2015 , and rebounded to $63.2 \%$ in 2016 .

Figure 2 presents the trends of susceptibility to ceftazidime-avibactam against key bacterial species over time in different regions using the CLSI 2019 breakpoint. The susceptibility of E. coli, K. pneumoniae, and P. mirabilis to ceftazidime-avibactam remained high (>95\%) and relatively stable over time, but with some decreases were observed in specific regions. The susceptibilities of E. cloacae and C. freundii to ceftazidime-avibactam remained relatively stable over time in all regions, but the susceptibilities in Asia (2013/2016: 94.6\%/94.6\% and $94.9 \% / 94.7 \%$ ) decreased in 2013 and were consistently lower than the global rates there after $(2013 / 2016$ : 98.3\%/97.4\% and 99.7\%/97.6\%). The global susceptibilities of $P$. aeruginosa to ceftazidime-avibactam globally decreased from 2012 to 2016 (2012/2016: 97.1\%/92.0\%), with lower rates observed in Latin America (2012/2016: 92.7\%/86.6\%), and higher rates observed in North America $(2012 / 2016$ : $97.9 \% / 96.6 \%)$.

\section{Global trend of the susceptibilities to ceftaroline and ceftazidime-avibactam against multi-drug-resistant species}

The proportion of methicillin-resistant S. aureus among all S. aureus remained stable from 2012 to 2016 (59.8\% in 2012 and 2016), with higher prevalence observed in North America (2012/2016: 66.5\%/68.1\%) and lower prevalence observed in Latin America (2012/2016: 
Table 2 In vitro susceptibilities of Gram-positive strains obtained from the ATLAS program, 2012-2016.

\begin{tabular}{|c|c|c|c|c|c|c|c|c|c|c|}
\hline \multirow[t]{2}{*}{ Organism/antibiotic } & \multirow[t]{2}{*}{ No. of isolates } & \multirow[t]{2}{*}{$\mathrm{MIC}_{50}$} & \multirow[t]{2}{*}{$\mathrm{MIC}_{90}$} & \multirow[t]{2}{*}{ MIC range } & \multicolumn{3}{|l|}{ CLSI } & \multicolumn{3}{|c|}{ EUCAST } \\
\hline & & & & & $\mathrm{S} \%$ & $1 \%$ & $\mathrm{R} \%$ & $\overline{S \%}$ & $1 \%$ & R\% \\
\hline \multicolumn{11}{|l|}{ Staphylococcus aureus } \\
\hline Ceftaroline & 50525 & 0.5 & 1 & $0.015-64$ & 93.4 & 6.2 & 0.4 & 93.4 & 6.2 & 0.4 \\
\hline Ceftazidime-avibactam & 50525 & 32 & 64 & $0.015-64$ & NA & NA & NA & NA & NA & NA \\
\hline Ceftazidime & 50525 & 32 & 64 & $0.015-64$ & NA & NA & NA & NA & NA & NA \\
\hline Pip-taz & 50525 & 8 & 32 & $0.12-32$ & NA & NA & NA & NA & NA & NA \\
\hline Levofloxacin & 50525 & 0.5 & 8 & $0.015-8$ & 56.9 & 0.4 & 42.7 & 56.9 & 0 & 43.1 \\
\hline Moxifloxacin & 50525 & 0.12 & 4 & $0.008-8$ & 57.1 & 2.7 & 40.1 & 56.8 & 0 & 43.2 \\
\hline Tigecycline & 50525 & 0.12 & 0.25 & $0.015-4$ & 98.9 & 1.1 & 0 & 98.9 & 0 & 1.1 \\
\hline Minocycline & 50525 & 0.12 & 1 & $0.12-16$ & 93.2 & 3.4 & 3.4 & 89.4 & 1.4 & 9.2 \\
\hline Gentamicin & 31019 & 0.5 & 64 & $0.06-64$ & 85 & 0.7 & 14.3 & 56.1 & 0 & 43.9 \\
\hline Daptomycin & 50525 & 0.5 & 1 & $0.06-4$ & 99.8 & 0.2 & 0 & 99.8 & 0 & 0.2 \\
\hline Trimethoprim sulfa & 31019 & 0.25 & 1 & $0.25-8$ & 96.8 & 0 & 3.3 & 96.8 & 0.7 & 2.6 \\
\hline Teicoplanin & 50525 & 0.5 & 1 & $0.12-32$ & 100 & 0 & 0 & 98.1 & 0 & 1.9 \\
\hline Vancomycin & 50525 & 1 & 2 & $0.25-4$ & 100 & 0 & 0 & 100 & 0 & 0 \\
\hline Clindamycin & 50525 & 0.12 & 4 & $0.03-8$ & 74.8 & 0.3 & 24.9 & 74.2 & 0.6 & 25.2 \\
\hline Erythromycin & 50525 & 1 & 16 & $0.12-16$ & 48 & 3.4 & 48.6 & 50.6 & 0.3 & 49.1 \\
\hline Linezolid & 50525 & 2 & 2 & $0.5-16$ & 100 & 0 & 0 & 100 & 0 & 0 \\
\hline Oxacillin & 50525 & 4 & 8 & $0.06-8$ & 40.4 & 0 & 59.6 & NA & NA & NA \\
\hline \multicolumn{11}{|l|}{ Streptococcus pneumoniae } \\
\hline Ceftaroline & 11005 & 0.008 & 0.12 & $0.004-32$ & 99.7 & 0.3 & 0 & 98.7 & 0 & 1.3 \\
\hline Ceftazidime-avibactam & 11005 & 0.25 & 16 & $0.015-128$ & NA & NA & NA & NA & NA & NA \\
\hline Ceftazidime & 11005 & 0.25 & 16 & $0.015-128$ & NA & NA & NA & NA & NA & NA \\
\hline Doripenem & 11005 & 0.015 & 1 & $0.015-8$ & 98 & 2 & 0 & 98 & 0 & 2 \\
\hline Meropenem & 11005 & 0.015 & 1 & $0.008-2$ & 78 & 9.2 & 12.8 & 100 & 0 & 0 \\
\hline Levofloxacin & 11005 & 1 & 1 & $0.12-16$ & 98.5 & 0.2 & 1.3 & 98.5 & 0 & 1.5 \\
\hline Moxifloxacin & 11005 & 0.12 & 0.25 & $0.03-8$ & 98.5 & 0.5 & 1.1 & 98.4 & 0 & 1.6 \\
\hline Tigecycline & 11005 & 0.03 & 0.03 & $0.008-2$ & 99.9 & 0.1 & 0 & NA & NA & NA \\
\hline Minocycline & 11005 & 0.12 & 4 & $0.015-4$ & 71.3 & 5.1 & 23.6 & 69.6 & 1.7 & 28.7 \\
\hline Daptomycin & 11005 & 0.25 & 0.5 & $0.03-8$ & NA & NA & NA & NA & NA & NA \\
\hline Vancomycin & 11005 & 0.25 & 0.5 & $0.008-2$ & 100 & 0 & 0 & 100 & 0 & 0 \\
\hline Clindamycin & 11005 & 0.06 & 2 & $0.008-2$ & 74.8 & 0.4 & 24.8 & 75.2 & 0 & 24.8 \\
\hline Erythromycin & 11005 & 0.06 & 2 & $0.008-2$ & 64.3 & 0.3 & 35.4 & 64.3 & 0.3 & 35.4 \\
\hline Linezolid & 11005 & 1 & 2 & $0.06-4$ & 100 & 0 & 0 & 100 & 0 & 0 \\
\hline Penicillin & 11005 & 0.03 & 2 & $0.015-16$ & 61.8 & 20.7 & 17.5 & 61.8 & 28.9 & 9.3 \\
\hline \multicolumn{11}{|l|}{ a-hemolytic Streptococcus } \\
\hline Ceftaroline & 12138 & 0.008 & 0.12 & $0.004-32$ & 99.7 & 0.3 & 0 & 98.7 & 0 & 1.3 \\
\hline Ceftazidime-avibactam & 12138 & 0.25 & 16 & $0.015-128$ & NA & NA & NA & NA & NA & NA \\
\hline Ceftazidime & 12138 & 0.25 & 16 & $0.015-128$ & NA & NA & NA & NA & NA & NA \\
\hline Penicillin & 12138 & 0.03 & 2 & $0.015-16$ & NA & NA & NA & NA & NA & NA \\
\hline Doripenem & 12138 & 0.015 & 1 & $0.015-8$ & NA & NA & NA & 98 & 0 & 2 \\
\hline Meropenem & 12138 & 0.015 & 1 & $0.008-2$ & NA & NA & NA & 100 & 0 & 0 \\
\hline Levofloxacin & 12138 & 1 & 2 & $0.12-16$ & 98.3 & 0.3 & 1.4 & 98.5 & 0 & 1.5 \\
\hline Moxifloxacin & 12138 & 0.12 & 0.25 & $0.03-8$ & 98.5 & 0.5 & 1.1 & 98.4 & 0 & 1.6 \\
\hline Minocycline & 12138 & 0.12 & 4 & $0.015-4$ & NA & NA & NA & 69.6 & 1.7 & 28.7 \\
\hline Tigecycline & 12138 & 0.03 & 0.03 & $0.008-2$ & NA & NA & NA & 100 & 0 & 0 \\
\hline Clindamycin & 12138 & 0.06 & 2 & $0.008-2$ & 75.6 & 0.4 & 24.1 & 75.9 & 0 & 24.1 \\
\hline Erythromycin & 12138 & 0.06 & 2 & $0.008-2$ & 64.8 & 0.3 & 34.9 & 64.3 & 0.3 & 35.4 \\
\hline Vancomycin & 12138 & 0.5 & 0.5 & $0.008-2$ & 100 & 0 & 0 & 100 & 0 & 0 \\
\hline
\end{tabular}


Table 2 (continued)

\begin{tabular}{|c|c|c|c|c|c|c|c|c|c|c|}
\hline \multirow[t]{2}{*}{ Organism/antibiotic } & \multirow[t]{2}{*}{ No. of isolates } & \multirow[t]{2}{*}{$\mathrm{MIC}_{50}$} & \multirow[t]{2}{*}{$\mathrm{MIC}_{90}$} & \multirow[t]{2}{*}{ MIC range } & \multicolumn{3}{|l|}{ CLSI } & \multicolumn{3}{|c|}{ EUCAST } \\
\hline & & & & & $\mathrm{S} \%$ & $1 \%$ & $\mathbf{R} \%$ & S\% & $1 \%$ & $\mathbf{R} \%$ \\
\hline Linezolid & 12138 & 1 & 2 & $0.06-8$ & 100 & 0 & 0 & 100 & 0 & 0 \\
\hline Daptomycin & 12138 & 0.25 & 0.5 & $0.03-8$ & 99.3 & 0.7 & 0 & 100 & 0 & 0 \\
\hline \multicolumn{11}{|l|}{$\beta$-hemolytic Streptococcus } \\
\hline Ceftaroline & 9019 & 0.004 & 0.015 & $0.004-1$ & 100 & 0 & 0 & NA & NA & NA \\
\hline Ceftazidime-avibactam & 9019 & 0.12 & 0.5 & $0.015-128$ & NA & NA & NA & NA & NA & NA \\
\hline Ceftazidime & 9019 & 0.12 & 0.5 & $0.015-128$ & NA & NA & NA & NA & NA & NA \\
\hline Penicillin & 9019 & 0.015 & 0.06 & $0.015-8$ & NA & NA & NA & NA & NA & NA \\
\hline Doripenem & 9019 & 0.015 & 0.03 & $0.015-8$ & NA & NA & NA & 100 & 0 & 0 \\
\hline Meropenem & 9019 & 0.015 & 0.06 & $0.008-2$ & 99.9 & 0.1 & 0 & 100 & 0 & 0 \\
\hline Levofloxacin & 9019 & 0.5 & 1 & $0.12-16$ & 98.3 & 0.2 & 1.5 & 98.2 & 0 & 1.9 \\
\hline Moxifloxacin & 9019 & 0.12 & 0.25 & $0.03-8$ & NA & NA & NA & 98.1 & 0 & 1.9 \\
\hline Minocycline & 9019 & 0.12 & 4 & $0.015-4$ & 69.9 & 30.1 & 0 & 65.6 & 0.9 & 33.4 \\
\hline Tigecycline & 9019 & 0.03 & 0.06 & $0.008-2$ & 100 & 0 & 0 & 100 & 0 & 0 \\
\hline Clindamycin & 9019 & 0.06 & 0.12 & $0.008-2$ & 90.6 & 0.3 & 9 & 91 & 0 & 9 \\
\hline Erythromycin & 9019 & 0.06 & 2 & $0.008-2$ & 83.4 & 0.7 & 15.8 & 84.4 & 0.6 & 15 \\
\hline Vancomycin & 9019 & 0.5 & 0.5 & $0.008-1$ & 100 & 0 & 0 & 100 & 0 & 0 \\
\hline Linezolid & 9019 & 1 & 2 & $0.06-8$ & 100 & 0 & 0 & 100 & 0 & 0 \\
\hline Daptomycin & 9019 & 0.12 & 0.5 & $0.03-8$ & 100 & 0 & 0 & 100 & 0 & 0 \\
\hline \multicolumn{11}{|l|}{ CONS } \\
\hline Ceftaroline & 8490 & 0.25 & 1 & $0.015-64$ & NA & NA & NA & NA & NA & NA \\
\hline Ceftazidime-avibactam & 8490 & 16 & 64 & $0.015-64$ & NA & NA & NA & NA & NA & NA \\
\hline Ceftazidime & 8490 & 16 & 64 & $0.015-64$ & NA & NA & NA & NA & NA & NA \\
\hline Pip-taz & 8490 & 2 & 32 & $0.12-32$ & NA & NA & NA & NA & NA & NA \\
\hline Levofloxacin & 8490 & 4 & 8 & $0.015-8$ & 46.9 & 1.8 & 51.4 & 46.9 & 0 & 53.1 \\
\hline Moxifloxacin & 8490 & 1 & 4 & $0.008-8$ & NA & NA & NA & NA & NA & NA \\
\hline Minocycline & 8490 & 0.25 & 0.5 & $0.12-16$ & 48.9 & 14.6 & 36.5 & 46.5 & 0 & 53.5 \\
\hline Tigecycline & 8490 & 0.25 & 0.5 & $0.015-4$ & 98.7 & 1.3 & 0 & 98.7 & 0 & 1.3 \\
\hline Clindamycin & 8490 & 0.12 & 8 & $0.03-8$ & 65.5 & 2.1 & 32.4 & 63.7 & 1.9 & 34.5 \\
\hline Erythromycin & 8490 & 8 & 16 & $0.12-16$ & 33.1 & 1.1 & 65.8 & 33.4 & 0.3 & 66.3 \\
\hline Vancomycin & 8490 & 1 & 2 & $0.25-8$ & 99.9 & 0.1 & 0 & 99.9 & 0 & 0.1 \\
\hline Teicoplanin & 8490 & 2 & 8 & $0.12-64$ & 98 & 1.7 & 0.3 & 85 & 0 & 15 \\
\hline Linezolid & 8490 & 1 & 2 & $0.5-16$ & 99.4 & 0 & 0.6 & 99.4 & 0 & 0.6 \\
\hline Daptomycin & 8490 & 0.5 & 1 & $0.06-4$ & 99.6 & 0.4 & 0 & 99.6 & 0 & 0.4 \\
\hline Gentamicin & 5336 & 2 & 64 & $0.06-64$ & 54.7 & 5.5 & 39.8 & 33.8 & 0 & 66.3 \\
\hline Trimethoprim sulfa & 5336 & 1 & 8 & $0.25-8$ & 61 & 0 & 39 & 61 & 10 & 28.9 \\
\hline Oxacillin & 8490 & 4 & 8 & $0.06-8$ & 25.7 & 0 & 74.3 & NA & NA & NA \\
\hline \multicolumn{11}{|l|}{ Enterococcus faecalis } \\
\hline Ceftaroline & 3194 & 1 & 16 & $0.015-64$ & NA & NA & NA & NA & NA & NA \\
\hline Ceftazidime-avibactam & 3194 & 64 & 64 & $1-64$ & NA & NA & NA & NA & NA & NA \\
\hline Ceftazidime & 3194 & 64 & 64 & $1-64$ & NA & NA & NA & NA & NA & NA \\
\hline Levofloxacin & 3194 & 1 & 16 & $0.06-16$ & 68 & 1.1 & 30.8 & NA & NA & NA \\
\hline Tigecycline & 3194 & 0.12 & 0.25 & $0.015-4$ & 94.1 & 5.9 & 0 & 94.1 & 3.9 & 2 \\
\hline Minocycline & 3194 & 16 & 16 & $0.06-16$ & 25.8 & 13.7 & 60.5 & NA & NA & NA \\
\hline Daptomycin & 3194 & 2 & 4 & $0.06-8$ & 99.8 & 0.2 & 0 & NA & NA & NA \\
\hline Teicoplanin & 3194 & 0.5 & 0.5 & $0.12-64$ & 98.3 & 0.1 & 1.7 & 97.8 & 0 & 2.2 \\
\hline Vancomycin & 3194 & 1 & 2 & $0.12-64$ & 94.3 & 3.8 & 1.9 & 94.3 & 0 & 5.7 \\
\hline Erythromycin & 3194 & 16 & 16 & $0.06-16$ & 14.6 & 27.4 & 58 & NA & NA & NA \\
\hline Linezolid & 3194 & 1 & 2 & $0.06-8$ & 99.3 & 0.6 & 0.2 & 99.8 & 0 & 0.2 \\
\hline
\end{tabular}


Table 2 (continued)

\begin{tabular}{|c|c|c|c|c|c|c|c|c|c|c|}
\hline \multirow[t]{2}{*}{ Organism/antibiotic } & \multirow[t]{2}{*}{ No. of isolates } & \multirow[t]{2}{*}{$\mathrm{MIC}_{50}$} & \multirow[t]{2}{*}{$\mathrm{MIC}_{90}$} & \multirow[t]{2}{*}{ MIC range } & \multicolumn{3}{|l|}{ CLSI } & \multicolumn{3}{|c|}{ EUCAST } \\
\hline & & & & & $\mathrm{S} \%$ & $1 \%$ & $\mathrm{R} \%$ & $\mathrm{~S} \%$ & $1 \%$ & $\mathrm{R} \%$ \\
\hline Quinupristin dalfopristin & 2014 & 8 & 16 & $0.25-16$ & 1 & 7.7 & 91.3 & NA & NA & NA \\
\hline \multicolumn{11}{|l|}{ Enterococcus faecium } \\
\hline Ceftaroline & 2546 & 64 & 64 & $0.03-64$ & NA & NA & NA & NA & NA & NA \\
\hline Ceftazidime-avibactam & 2546 & 64 & 64 & $0.12-64$ & NA & NA & NA & NA & NA & NA \\
\hline Ceftazidime & 2546 & 64 & 64 & $0.12-64$ & NA & NA & NA & NA & NA & NA \\
\hline Levofloxacin & 2546 & 16 & 16 & $0.06-16$ & 12.2 & 3.9 & 83.9 & NA & NA & NA \\
\hline Tigecycline & 2546 & 0.12 & 0.25 & $0.015-8$ & 95.5 & 4.5 & 0 & 95.5 & 3 & 1.5 \\
\hline Minocycline & 2546 & 2 & 16 & $0.06-16$ & 55.4 & 12.4 & 32.2 & NA & NA & NA \\
\hline Daptomycin & 2546 & 4 & 4 & $0.06-16$ & 98 & 2 & 0 & NA & NA & NA \\
\hline Teicoplanin & 2546 & 1 & 64 & $0.12-64$ & 76 & 1 & 23 & 75.2 & 0 & 24.8 \\
\hline Vancomycin & 2546 & 1 & 64 & $0.12-64$ & 69.2 & 5 & 25.8 & 69.2 & 0 & 30.8 \\
\hline Erythromycin & 2546 & 16 & 16 & $0.06-16$ & 3.6 & 11.2 & 85.2 & NA & NA & NA \\
\hline Linezolid & 2546 & 1 & 2 & $0.06-16$ & 97.4 & 2.5 & 0.2 & 99.8 & 0 & 0.2 \\
\hline Quinupristin dalfopristin & 1577 & 1 & 4 & $0.06-16$ & 73.2 & 13.9 & 12.9 & NA & NA & NA \\
\hline
\end{tabular}

CLSI Clinical Laboratory and Standards Institute, EUCAST European Committee on Antimicrobial Susceptibility Testing, NA not applicable, CoNS coagulase-negative staphylococci

$55.9 \% / 53.3 \%)$. The overall global susceptibility of methicillin-resistant $S$. aureus to ceftaroline increased slightly from $87.5 \%$ in 2012 to $91.7 \%$ in 2016 , with a marked increase observed in Africa-Middle East (2012/2016: 88.7\%/97.8\%), Europe (2012/2016: 89.8\%/96.2\%), and Latin America (2012/2016: 78.2\%/88.2\%) (Fig. 3a). The susceptibility of methicillin-resistant $S$. aureus to ceftaroline in Asia was consistently lower than in all other regions (2012/2016: 75.2\%/75.5\%).

The proportion of CR-K. pneumonia among all K. pneumoniae lightly increased from $6.7 \%$ in 2012 to8.2\% in 2016, with higher prevalence observed in Latin America (2012/2016: 9.2\%/11.2\%) and Europe (2012/2016: 9.3\%/10.4\%). Conversely, the overall global susceptibility of CR-K. pneumoniae to ceftazidimeavibactam decreased from $88.4 \%$ in 2012 to $81.6 \%$ in 2016, with a marked decrease observed in Africa-Middle East (2012/2016: 100\%/63.6\%), Asia (2012/2016: 76.9\%/68.2\%), and Latin America (2012/2016: $100 \% / 90 \%$ ) (Fig. 3b). The susceptibility rates in Asia and Africa-Middle East were, in general, lower than in the other regions during the study period.

The proportion of CR-P. aeruginosa among all $P$. aeruginosa remained relatively stable over time (2012/2016: 26.5\%/26.7\%), with higher prevalence observed for Latin America (2012/2016: 36.3\%/34.4\%). The overall global susceptibility of CR-P. aeruginosa to ceftazidime-avibactam decreased from $89.6 \%$ in 2012 to $72.7 \%$ in 2016 , with a marked decrease observed for all regions (Fig. 3). The susceptibility rate in North
America (2012/2016: 93.2\%/86.0\%) was, in general, higher than in other regions.

\section{Discussion}

Ceftaroline and ceftazidime-avibactam are relatively recent antibiotics that are active against a variety of bacterial species, including some with innate antibiotic resistance $[10-13,15]$. The exact resistance patterns to those antibiotics still need to be defined exactly, and there is a crucial need for global surveillance of antibiotic resistance. This study reveals the patterns of the susceptibilities of different bacterial species to a variety of antibiotics, with a focus on ceftaroline and ceftazidime-avibactam, around the world, and over 5 years. The results indicate that the global resistance of CR-P. aeruginosa to ceftazidime-avibactam greatly increased over time, while the susceptibility profile of ceftaroline and ceftazidime-avibactam against other species were relatively stable.

The first objective of this study was to examine the overall in vitro activities of ceftaroline and ceftazidime-avibactam using data from the ATLAS program. The results showed that ceftaroline was highly potent ( $>90 \%$ susceptibility) against Gram-positive strains, including S. aureus, S. pneumoniae, and Streptococcus. On the other hand, ceftazidime-avibactam showed susceptibility $>90 \%$ against Gram-negative bacteria, including Enterobacteriaceae, P. aeruginosa, and $P$. mirabilis, with overtly increased antimicrobial activity observed with the addition of avibactam to 
Table 3 In vitro susceptibilities of multi-drug resistant strains obtained from the ATLAS program, 2012-2016.

\begin{tabular}{|c|c|c|c|c|c|c|c|c|c|c|}
\hline \multirow[t]{2}{*}{ Organism/antibiotic } & \multirow[t]{2}{*}{ No. of isolates } & \multirow[t]{2}{*}{$\mathrm{MIC}_{50}$} & \multirow[t]{2}{*}{$\mathrm{MIC}_{90}$} & \multirow[t]{2}{*}{ MIC range } & \multicolumn{3}{|l|}{$C L S I^{a}$} & \multicolumn{3}{|c|}{ EUCAST } \\
\hline & & & & & $\mathrm{S} \%$ & $1 \%$ & $\mathrm{R} \%$ & $\mathrm{~S} \%$ & $1 \%$ & $\mathrm{R} \%$ \\
\hline \multicolumn{11}{|l|}{ CRECO } \\
\hline Ceftaroline & 119 & 256 & 256 & $0.015-256$ & 10.9 & 1.7 & 87.4 & 9.5 & 0 & 90.5 \\
\hline Ceftazidime-avibactam & 119 & 0.5 & 256 & $0.03-256$ & 72.3 & 0 & 27.7 & 40.5 & 0 & 59.5 \\
\hline Ceftazidime & 119 & 64 & 256 & $0.12-256$ & 22.7 & 4.2 & 73.1 & 11.9 & 2.4 & 85.7 \\
\hline Cefepime & 119 & 32 & 32 & $0.12-32$ & 8.4 & 16 & 75.6 & 0 & 2.4 & 97.6 \\
\hline Pip-taz & 119 & 256 & 256 & $0.5-256$ & 21.9 & 5.9 & 72.3 & 19.1 & 2.4 & 78.6 \\
\hline Doripenem & 119 & 4 & 16 & $0.03-16$ & 32.8 & 11.8 & 55.5 & 19.1 & 2.4 & 78.6 \\
\hline Imipenem & 119 & 8 & 16 & $4-16$ & 0 & 0 & 100 & 0 & 0 & 100 \\
\hline Meropenem & 119 & 8 & 16 & $0.015-16$ & 26.1 & 6.7 & 67.2 & 19.1 & 7.1 & 73.8 \\
\hline Levofloxacin & 119 & 8 & 16 & $0.015-16$ & 24.4 & 3.4 & 72.3 & 7.1 & 2.4 & 90.5 \\
\hline Tigecycline & 119 & 0.25 & 1 & $0.03-4$ & 98.3 & 1.7 & 0 & 88.1 & 7.1 & 4.8 \\
\hline Amikacin & 119 & 8 & 64 & $1-64$ & 78.2 & 5 & 16.8 & 59.5 & 7.1 & 33.3 \\
\hline Colistin & 79 & 0.5 & 1 & $0.12-16$ & NA & NA & NA & 96.3 & 0 & 3.7 \\
\hline Aztreonam & 119 & 64 & 256 & $0.015-256$ & 23.5 & 0.8 & 75.6 & 16.7 & 2.4 & 81 \\
\hline \multicolumn{11}{|l|}{ CRKPN } \\
\hline Ceftaroline & 1418 & 256 & 256 & $0.06-256$ & 0.6 & 0 & 99.4 & 0 & 0 & 100 \\
\hline Ceftazidime-avibactam & 1418 & 1 & 256 & $0.015-256$ & 85.6 & 0 & 14.4 & 83.6 & 0 & 16.4 \\
\hline Ceftazidime & 1418 & 256 & 256 & $0.12-256$ & 4 & 2.5 & 93.5 & 0.6 & 0.7 & 98.7 \\
\hline Cefepime & 1418 & 32 & 32 & $0.12-32$ & 3.5 & 8 & 88.6 & 0.4 & 0.6 & 99 \\
\hline Pip-taz & 1418 & 256 & 256 & $2-256$ & 1.5 & 1.1 & 97.4 & 0.3 & 0.1 & 99.6 \\
\hline Doripenem & 1418 & 8 & 16 & $0.03-16$ & 4.2 & 5.4 & 90.4 & 0.7 & 0.4 & 98.9 \\
\hline Imipenem & 1418 & 16 & 16 & $4-16$ & 0 & 0 & 100 & 0 & 0 & 100 \\
\hline Meropenem & 1418 & 16 & 16 & $0.015-16$ & 2.9 & 4 & 93.1 & 0.8 & 3.9 & 95.3 \\
\hline Levofloxacin & 1418 & 8 & 16 & $0.03-16$ & 12.7 & 3.5 & 83.9 & 3.1 & 1.6 & 95.2 \\
\hline Tigecycline & 1418 & 1 & 2 & $0.06-16$ & 92.6 & 6.3 & 1.1 & 74.7 & 17.1 & 8.2 \\
\hline Amikacin & 1418 & 16 & 64 & $0.25-64$ & 52.1 & 28.1 & 19.8 & 30.3 & 13.2 & 56.6 \\
\hline Colistin & 1046 & 1 & 16 & $0.06-16$ & NA & NA & NA & 74.2 & 0 & 25.8 \\
\hline Aztreonam & 1418 & 256 & 256 & $0.03-256$ & 4.4 & 0.4 & 95.1 & 2.7 & 0.2 & 97.1 \\
\hline \multicolumn{11}{|l|}{ CRECL } \\
\hline Ceftaroline & 149 & 256 & 256 & $0.06-256$ & 4.7 & 0.7 & 94.6 & 1.6 & 0 & 98.4 \\
\hline Ceftazidime-avibactam & 149 & 128 & 256 & $0.06-256$ & 42.3 & 0 & 57.7 & 21.9 & 0 & 78.1 \\
\hline Ceftazidime & 149 & 256 & 256 & $0.12-256$ & 8.7 & 1.3 & 89.9 & 1.6 & 1.6 & 96.9 \\
\hline Cefepime & 149 & 32 & 32 & $0.12-32$ & 14.8 & 10.7 & 74.5 & 4.7 & 0 & 95.3 \\
\hline Pip-taz & 149 & 256 & 256 & $2-256$ & 10.1 & 5.4 & 84.6 & 1.6 & 1.6 & 96.9 \\
\hline Doripenem & 149 & 8 & 16 & $0.06-16$ & 14.1 & 6.7 & 79.2 & 0 & 0 & 100 \\
\hline Imipenem & 149 & 8 & 16 & $4-16$ & 0 & 0 & 100 & 0 & 0 & 100 \\
\hline Meropenem & 149 & 8 & 16 & $0.03-16$ & 14.1 & 12.1 & 73.8 & 1.6 & 17.2 & 81.3 \\
\hline Levofloxacin & 149 & 4 & 16 & $0.03-16$ & 43.6 & 7.4 & 49 & 17.2 & 4.7 & 78.1 \\
\hline Tigecycline & 149 & 1 & 4 & $0.12-8$ & 83.9 & 14.1 & 2 & 59.4 & 15.6 & 25 \\
\hline Amikacin & 149 & 4 & 64 & $0.5-64$ & 80.5 & 5.4 & 14.1 & 60.9 & 7.8 & 31.3 \\
\hline Colistin & 118 & 0.5 & 1 & $0.12-16$ & NA & NA & NA & 93.8 & 0 & 6.3 \\
\hline Aztreonam & 149 & 64 & 256 & $0.06-256$ & 24.2 & 2 & 73.8 & 18.8 & 6.3 & 75 \\
\hline \multicolumn{11}{|l|}{ CRPAE } \\
\hline Ceftaroline & 4546 & 128 & 256 & $0.015-256$ & NA & NA & NA & NA & NA & NA \\
\hline Ceftazidime-avibactam & 4546 & 4 & 64 & $0.015-256$ & 74.5 & 0 & 25.5 & 70.6 & 0 & 29.4 \\
\hline Ceftazidime & 4546 & 16 & 128 & $0.12-256$ & 46.6 & 8.2 & 45.3 & 41.4 & 0 & 58.6 \\
\hline Cefepime & 4546 & 16 & 32 & $0.25-32$ & 47.2 & 23.2 & 29.6 & 41.5 & 0 & 58.6 \\
\hline Pip-taz & 4546 & 64 & 256 & $0.25-256$ & 34.7 & 24.8 & 40.5 & 29.4 & 0 & 70.7 \\
\hline
\end{tabular}


Table 3 (continued)

\begin{tabular}{|c|c|c|c|c|c|c|c|c|c|c|}
\hline \multirow[t]{2}{*}{ Organism/antibiotic } & \multirow[t]{2}{*}{ No. of isolates } & \multirow[t]{2}{*}{$\mathrm{MIC}_{50}$} & \multirow[t]{2}{*}{$\mathrm{MIC}_{90}$} & \multirow[t]{2}{*}{ MIC range } & \multicolumn{3}{|c|}{$\mathrm{CLSI}^{\mathrm{a}}$} & \multicolumn{3}{|c|}{ EUCAST } \\
\hline & & & & & $\mathrm{S} \%$ & $1 \%$ & $\mathrm{R} \%$ & $\mathrm{~S} \%$ & $1 \%$ & $\mathrm{R} \%$ \\
\hline Doripenem & 4545 & 8 & 16 & $0.03-16$ & 15.1 & 22.8 & 62.1 & 1.8 & 5.9 & 92.3 \\
\hline Imipenem & 4546 & 16 & 16 & $8-16$ & 0 & 0 & 100 & 0 & 0 & 100 \\
\hline Meropenem & 4546 & 16 & 16 & $0.06-16$ & 11.7 & 15.4 & 72.9 & 4.9 & 33.5 & 61.7 \\
\hline Levofloxacin & 4546 & 8 & 16 & $0.015-16$ & 36 & 10 & 54 & 22.1 & 0 & 77.9 \\
\hline Tigecycline & 4546 & 16 & 16 & $0.03-16$ & NA & NA & NA & NA & NA & NA \\
\hline Amikacin & 4546 & 8 & 64 & $0.25-64$ & 72.8 & 7.3 & 19.9 & 60.5 & 9.2 & 30.4 \\
\hline Colistin & 3521 & 1 & 2 & $0.06-16$ & 96.4 & 0 & 3.6 & 96.6 & 0 & 3.4 \\
\hline Aztreonam & 4546 & 16 & 128 & $0.06-256$ & 30.7 & 21 & 48.3 & 1.1 & 46.6 & 52.4 \\
\hline \multicolumn{11}{|l|}{ CRABA } \\
\hline Ceftaroline & 2318 & 256 & 256 & $2-256$ & NA & NA & NA & NA & NA & NA \\
\hline Ceftazidime-avibactam & 2318 & 64 & 256 & $0.06-256$ & NA & NA & NA & NA & NA & NA \\
\hline Ceftazidime & 2318 & 128 & 256 & $1-256$ & 5.1 & 2.3 & 92.7 & NA & NA & NA \\
\hline Cefepime & 2318 & 32 & 32 & $0.25-32$ & 3.6 & 10.7 & 85.7 & NA & NA & NA \\
\hline Pip-taz & 2318 & 256 & 256 & $4-256$ & 0.4 & 0.7 & 99 & NA & NA & NA \\
\hline Doripenem & 2318 & 8 & 16 & $0.5-16$ & 0.2 & 0.4 & 99.4 & 0 & 0 & 100 \\
\hline Imipenem & 2318 & 16 & 16 & $8-16$ & 0 & 0 & 100 & 0 & 0 & 100 \\
\hline Meropenem & 2318 & 16 & 16 & $1-16$ & 0.1 & 0.5 & 99.4 & 0 & 1.9 & 98.1 \\
\hline Levofloxacin & 2318 & 8 & 16 & $0.06-16$ & 3.8 & 11.4 & 84.9 & 1.4 & 0.6 & 98 \\
\hline Tigecycline & 2318 & 1 & 4 & $0.03-16$ & NA & NA & NA & NA & NA & NA \\
\hline Amikacin & 2318 & 64 & 64 & $0.25-64$ & 19.3 & 7.8 & 73 & 16.4 & 2.7 & 80.9 \\
\hline Colistin & 1552 & 1 & 2 & $0.12-16$ & 92.1 & 0 & 7.9 & 92 & 0 & 8 \\
\hline Aztreonam & 2318 & 64 & 256 & $2-256$ & NA & NA & NA & NA & NA & NA \\
\hline \multicolumn{11}{|l|}{ MRSA } \\
\hline Ceftaroline & 30100 & 0.5 & 2 & $0.03-64$ & 89.0 & 10.3 & 0.7 & NA & NA & NA \\
\hline Ceftazidime-avibactam & 30100 & 64 & 64 & $2-64$ & NA & NA & NA & NA & NA & NA \\
\hline Ceftazidime & 30100 & 64 & 64 & $1-64$ & NA & NA & NA & NA & NA & NA \\
\hline Pip-taz & 30100 & 32 & 32 & $0.12-32$ & NA & NA & NA & NA & NA & NA \\
\hline Doripenem & 30100 & 2 & 8 & $0.008-8$ & NA & NA & NA & NA & NA & NA \\
\hline Meropenem & 30100 & 4 & 16 & $0.015-16$ & NA & NA & NA & NA & NA & NA \\
\hline Levofloxacin & 30100 & 4 & 8 & $0.015-8$ & 32.4 & 0.5 & 67.1 & NA & NA & NA \\
\hline Moxifloxacin & 30100 & 2 & 4 & $0.008-8$ & 32.6 & 3.9 & 63.5 & NA & NA & NA \\
\hline Minocycline & 30100 & 0.12 & 8 & $0.12-16$ & 89.4 & 5.3 & 5.4 & NA & NA & NA \\
\hline Tigecycline & 30100 & 0.12 & 0.5 & $0.015-4$ & 98.5 & 1.5 & 0 & NA & NA & NA \\
\hline Clindamycin & 30100 & 0.12 & 8 & $0.03-8$ & 61 & 0.3 & 38.7 & NA & NA & NA \\
\hline Erythromycin & 30100 & 8 & 16 & $0.12-16$ & 29.7 & 2.5 & 67.8 & NA & NA & NA \\
\hline Vancomycin & 30100 & 1 & 2 & $0.25-4$ & 100 & 0 & 0 & NA & NA & NA \\
\hline Teicoplanin & 30100 & 1 & 2 & $0.12-32$ & 100 & 0 & 0 & NA & NA & NA \\
\hline Linezolid & 30100 & 2 & 2 & $0.5-16$ & 100 & 0 & 0 & NA & NA & NA \\
\hline Daptomycin & 30100 & 0.5 & 1 & $0.06-4$ & 99.7 & 0.3 & 0 & NA & NA & NA \\
\hline Gentamicin & 18616 & 1 & 64 & $0.06-64$ & 78.2 & 0.9 & 21 & NA & NA & NA \\
\hline Trimethoprim sulfa & 18616 & 0.25 & 1 & $0.25-8$ & 95.6 & 0 & 4.4 & NA & NA & NA \\
\hline Oxacillin & 30100 & 4 & 8 & $4-8$ & 0 & 0 & 100 & NA & NA & NA \\
\hline \multicolumn{11}{|l|}{ PRSP } \\
\hline Ceftaroline & 1925 & 0.12 & 0.25 & $0.008-32$ & 98.2 & 1.8 & 0 & 86.8 & 0 & 13.2 \\
\hline Ceftazidime-avibactam & 1925 & 16 & 64 & $1-128$ & NA & NA & NA & NA & NA & NA \\
\hline Ceftazidime & 1925 & 16 & 64 & $1-128$ & NA & NA & NA & NA & NA & NA \\
\hline Penicillin & 1925 & 4 & 8 & $2-16$ & 0 & 0 & 100 & 0 & 0 & 100 \\
\hline Doripenem & 1925 & 1 & 2 & $0.015-8$ & 89.4 & 10.6 & 0 & 81.1 & 0 & 18.9 \\
\hline
\end{tabular}


Table 3 (continued)

\begin{tabular}{|c|c|c|c|c|c|c|c|c|c|c|}
\hline \multirow[t]{2}{*}{ Organism/antibiotic } & \multirow[t]{2}{*}{ No. of isolates } & \multirow[t]{2}{*}{$\mathrm{MIC}_{50}$} & \multirow[t]{2}{*}{$\mathrm{MIC}_{90}$} & \multirow[t]{2}{*}{ MIC range } & \multicolumn{3}{|c|}{$\mathrm{CLSI}^{\mathrm{a}}$} & \multicolumn{3}{|c|}{ EUCAST } \\
\hline & & & & & $\mathrm{S} \%$ & $1 \%$ & $\mathbf{R} \%$ & $\mathrm{~S} \%$ & $1 \%$ & $\mathrm{R} \%$ \\
\hline Meropenem & 1925 & 1 & 2 & $0.008-2$ & 3.4 & 32.3 & 64.3 & 100 & 0 & 0 \\
\hline Levofloxacin & 1925 & 1 & 2 & $0.12-16$ & 95.3 & 0.5 & 4.2 & 94.1 & 0 & 6 \\
\hline Moxifloxacin & 1925 & 0.12 & 0.25 & $0.03-8$ & 95.7 & 1.5 & 2.8 & 94.6 & 0 & 5.4 \\
\hline Minocycline & 1925 & 4 & 4 & $0.03-4$ & 28.5 & 13.2 & 58.3 & 18.8 & 3 & 78.2 \\
\hline Tigecycline & 1925 & 0.03 & 0.03 & $0.008-2$ & 99.8 & 0.2 & 0 & NA & NA & NA \\
\hline Clindamycin & 1925 & 2 & 2 & $0.008-2$ & 32.6 & 0.3 & 67.2 & 24.3 & 0 & 75.7 \\
\hline Erythromycin & 1925 & 2 & 2 & $0.008-2$ & 13.9 & 0.2 & 86 & 10.9 & 0.1 & 89 \\
\hline Vancomycin & 1925 & 0.5 & 0.5 & $0.015-2$ & 99.9 & 0.1 & 0 & 100 & 0 & 0 \\
\hline Linezolid & 1925 & 1 & 1 & $0.06-2$ & 100 & 0 & 0 & 100 & 0 & 0 \\
\hline
\end{tabular}

CLSI Clinical Laboratory and Standards Institute, EUCAST European Committee on Antimicrobial Susceptibility Testing, CRECO Carbapenem-resistant Escherichia coli, CRKPN Carbapenem-resistant Klebsiella pneumonia, CRECL Carbapenem-resistant Enterobacter cloacae, CRPAE Carbapenem-resistant Pseudomonas aeruginosa, CRABA Carbapenem-resistant Acinetobacter baumannii, MRSA Methicillin-resistant Staphylococcus aureus, PRSP Penicillin-resistant Streptococcus pneumonia, NA not applicable

${ }^{a}$ Cefepime CLSI susceptibility for Enterobacteriaceae adopted the susceptible, susceptible-dose-dependent, and resistant categories

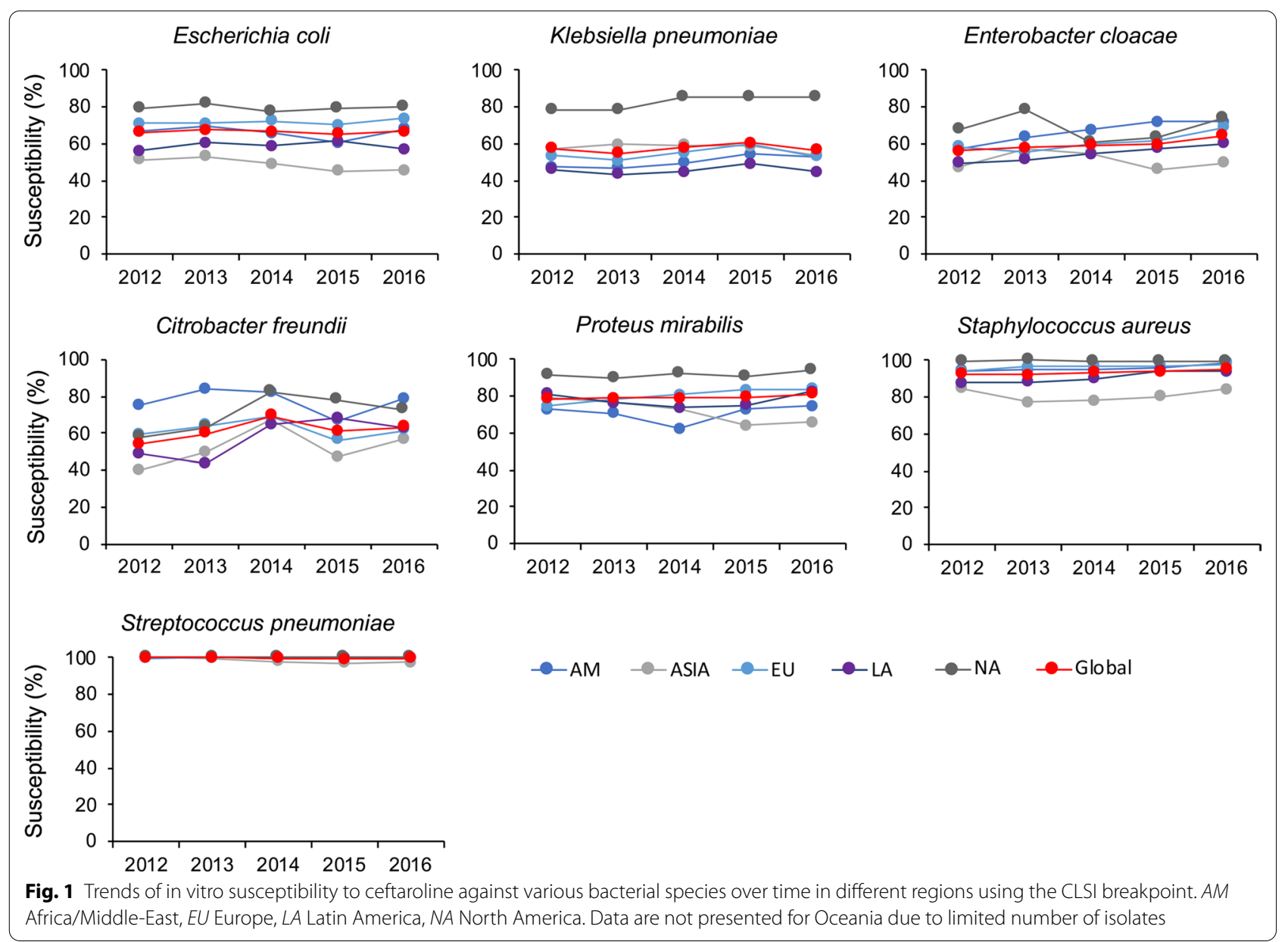

ceftazidime. Further analysis of the data from China showed that similar to the global pattern, the susceptibilities of E. coli, K. pneumoniae, and P. aeruginosa to ceftazidime-avibactam were high $(92.9-99.0 \%)$ in China. Those results are generally similar with those of surveillance studies in China [18], Asia [19], the United 

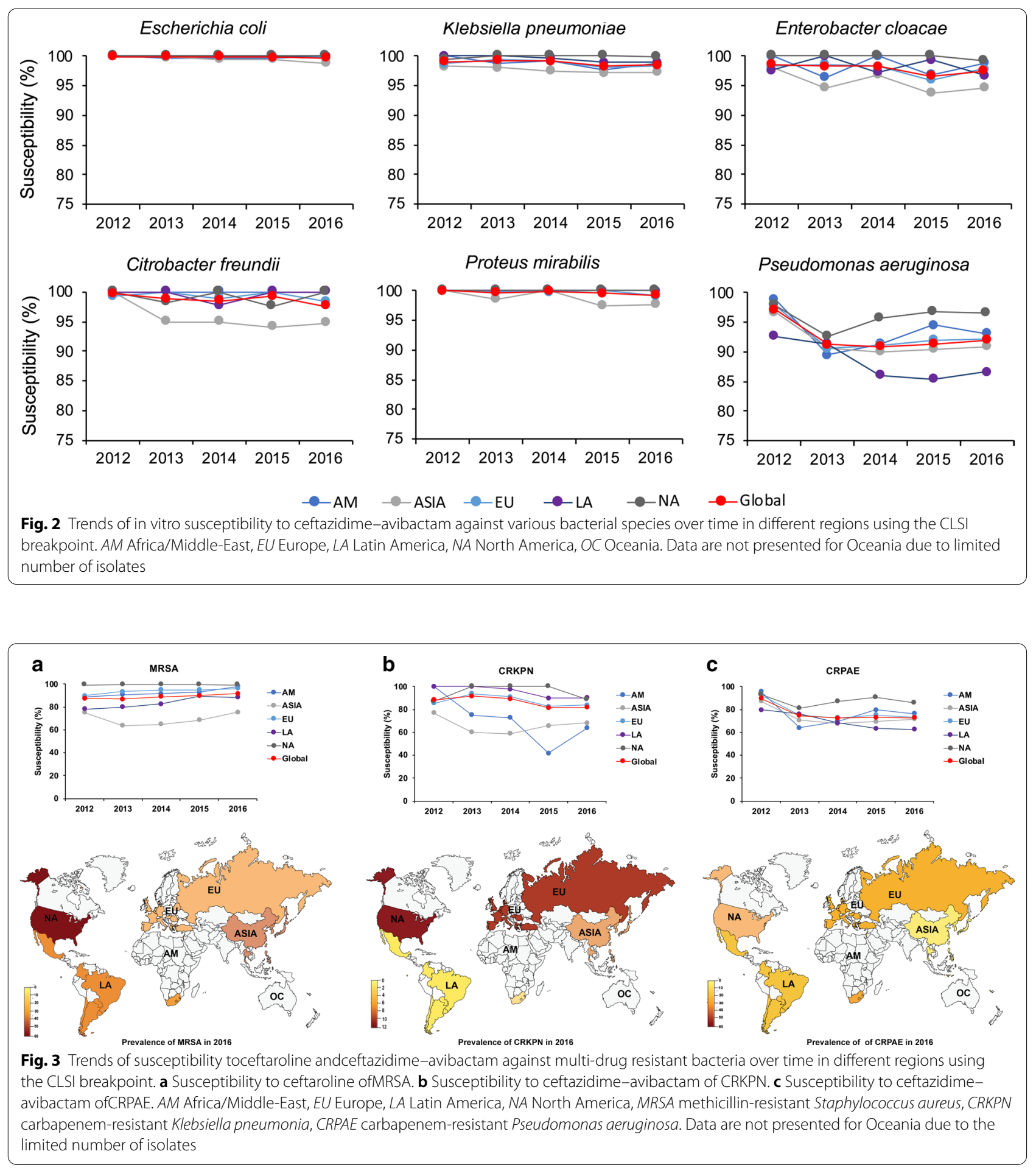

States of America [20-22], and Europe [23], and with the AWARE surveillance program [24-26], but with some minute differences that could be due to the specimens' area of origin since the present study included specimens from all over the world. Another source of difference could be the tested period since bacterial susceptibility changes over time.

Indeed, as shown by the results to the second objective of the present study, the patterns of resistance varied among species, among world regions, and over time. 
The main differences were that the susceptibility rates of E. coli and S. aureus to ceftaroline in Asia were lower than the global rates, while those in Europe and North America were generally similar or higher than the global rates. Asia also showed lower susceptibility rates to ceftazidime-avibactam against C. freundii, E. cloacae, and P. mirabilis. A study examined the resistance patterns to ceftaroline, ceftazidime, and piperacillin-tazobactam and revealed similar patterns between Europe and the United States of America [20]. A study across different areas of the United States of America also reported good susceptibility profiles of ceftaroline against respiratory pathogens [27]. A recent report from the World Health Organization revealed high rates of antibiotic resistance all over the world [28, 29]. Antibiotic resistance is a major concern worldwide, and significant differences in the resistance patterns can be observed. The World Health Organization highlighted that even if antibiotic resistance has increased all over the world, the increase was particularly alarming in Asia because of poor health and environment practices such as antibiotic over-prescription, poor infection control, poor waste management, overuse of antibiotics in farming, food security, and restricted access to the newest antibiotics [30-32]. Furthermore, the Asia-Pacific region is the most populous region in the world. Many of its countries are among the poorest, and poor health infrastructure is often encountered [33]. In addition, specific resistance mechanisms (e.g., the New Delhi metallo- $\beta$-lactamase-1) are also encountered in Asia [34]. The TEST study showed that Africa and Asia were the two regions of the world with the highest occurrence of $S$. aureus resistant to multiple antibiotics among blood-borne infections [35].

There is a plea for worldwide, automated, and comprehensive surveillance of antimicrobial resistance patterns $[8,36,37]$. Such surveillance could help optimize the worldwide use of antibiotics to improve infection control and minimize the occurrence of resistant strains [38]. In fact, surveillance and proper actions are necessary to avoid medical, social, and economic setbacks that could threaten the very fabric of the global community [38]. Even if the present study focused on ceftaroline and ceftazidime-avibactam, the ATLAS program provides the comprehensive global susceptibility profiles of many antibiotics against a large number of bacterial species. ATLAS receives data from all regions of the world and covers many years. Therefore, it helps provide certain help for the global surveillance of bacterial resistance.

This study has limitations. First, this was a retrospective study, with the inevitable confounding biases, such as the nature of the participating hospitals (mostly tertiary university-affiliated centers), the exact patient populations consulting at those hospitals, and the lack of many variables at the patient level. Second, this study is purely descriptive. Because of the large sample size, minute nonclinically significant differences in susceptibility could be statistically significant, which could be misleading [39, 40]; therefore, statistical tests were not performed.

\section{Conclusion}

In summary, the present study showed that the addition of avibactam improved the activity of ceftazidime against Enterobacteriaceae and $P$. aeruginosa. The global antimicrobial susceptibilitiestoceftaroline and ceftazidime-avibactam were, in general, stable from 2012 to 2016, but a marked reduction in the susceptibilities of specific species and CR-P. aeruginosa for ceftazidime-avibactam was observed in specific regions of the world.

\section{Acknowledgements \\ We are grateful to IHMA laboratory staff for their work on the antimicrobial susceptibility tests.}

\section{Authors' contributions}

$H Z, Y C X, P Y J$, and QWY conceived and designed the study, performed the experiments, analyzed the data, and wrote the paper. YZ, GZ, JJZ, WK, SMD, TW, RJ, JWC, and YLL helped perform the experiments. All authors read and approved the final manuscript.

\section{Funding}

This study was supported by Pfizer Inc. Further support was provided by the National Key Research and Development Program of China (2018YFC1200100, 2018YFC1200105) and CAMS Initiative for Innovative Medicine (Grant No. 2016-I2M-3-014). The funders had no role in the study design, collection, and analysis of data, interpretation of results, or preparation of the manuscript.

\section{Competing interests}

All authors declare that they have no conflict of interest and have submitted the ICMJE Form for disclosure of potential conflicts of interest. Conflicts that the editors consider relevant to the content of the manuscript have been disclosed.

Received: 28 May 2020 Accepted: 15 October 2020

Published online: 27 October 2020

\section{References}

1. Khameneh B, Diab R, Ghazvini K, Fazly Bazzaz BS. Breakthroughs in bacterial resistance mechanisms and the potential ways to combat them. Microb Pathog. 2016;95:32-42.

2. Chellat MF, Raguz L, Riedl R. Targeting antibiotic resistance. Angew Chem. 2016;55(23):6600-26.

3. Siddiqui $\mathrm{AH}$, Koirala J. Methicillin resistant Staphylococcus aureus (MRSA). Treasure Island: StatPearls; 2019

4. Akova M. Epidemiology of antimicrobial resistance in bloodstream infections. Virulence. 2016;7(3):252-66.

5. Tacconelli E, Carrara E, Savoldi A, et al. Discovery, research, and development of new antibiotics: the WHO priority list of antibiotic-resistant bacteria and tuberculosis. Lancet Infect Dis. 2018;18(3):318-27.

6. Chipolombwe J, Torok ME, Mbelle N, Nyasulu P. Methicillin-resistant Staphylococcus aureus multiple sites surveillance: a systemic review of the literature. Infect Drug Resist. 2016;9:35-42.

7. Frieri M, Kumar K, Boutin A. Antibiotic resistance. J Infect Public Health. 2017;10(4):369-78.

8. Fuhrmeister AS, Jones RN. The importance of antimicrobial resistance monitoring worldwide and the origins of SENTRY antimicrobial surveillance program. Open Forum Infect Dis. 2019;6(Suppl 1):S1-4. 
9. Duplessis C, Crum-Cianflone NF. Ceftaroline: a new cephalosporin with activity against methicillin-resistant Staphylococcus aureus (MRSA). Clin Med Rev Ther. 2011;3:1-17.

10. El Hajj MS, Turgeon RD, Wilby KJ. Ceftaroline fosamil for communityacquired pneumonia and skin and skin structure infections: a systematic review. Int J Clin Pharm. 2017;39(1):26-32.

11. Pawluk SA, Wilby KJ. Ceftaroline fosamil for community-acquired pneumonia. Lancet Infect Dis. 2015;15(9):999.

12. Carreno JJ, Lodise TP. Ceftaroline fosamil for the treatment of community-acquired pneumonia: from focus to capture. Infect Dis Ther. 2014;3(2):123-32

13. Mpenge MA, MacGowan AP. Ceftaroline in the management of complicated skin and soft tissue infections and community acquired pneumonia. Ther Clin Risk Manag. 2015;11:565-79.

14. Ehmann DE, Jahic H, Ross PL, et al. Avibactam is a covalent, reversible, non-beta-lactam beta-lactamase inhibitor. Proc Natl Acad Sci USA. 2012;109(29):11663-8.

15. Shirley M. Ceftazidime-avibactam: a review in the treatment of serious gram-negative bacterial infections. Drugs. 2018;78(6):675-92.

16. Clinical and Laboratory Standards Institute (CLSI). Performance standards for antimicrobial susceptibility testing. 29th ed. CLSI supplement M100. Wayne, PA: CLSI; 2019.

17. The European Committee on Antimicrobial Susceptibility Testing (EUCAST). Breakpoint tables for interpretation of MICs and zone diameters. Version 9.0. 2019. https://www.eucast.org/clinical_breakpoints/. Accessed 2 Jan 2019.

18. Zhou M, Chen J, Liu Y, et al. In vitro activities of Ceftaroline/Avibactam, Ceftazidime/Avibactam, and other comparators against pathogens from various complicated infections in China. Clin Infect Dis. 2018;67(suppl_2):S206-16.

19. Karlowsky JA, Kazmierczak KM, Bouchillon SK, de Jonge BLM, Stone GG, Sahm DF. In vitro activity of ceftazidime-avibactam against clinical isolates of enterobacteriaceae and Pseudomonas aeruginosa collected in Asia-Pacific countries: results from the INFORM Global Surveillance Program, 2012 to 2015. Antimicrob Agents Chemother. 2018; 62(7):e02569-17.

20. Jones RN, Farrell DJ, Mendes RE, Sader HS. Comparative ceftaroline activity tested against pathogens associated with community-acquired pneumonia: results from an international surveillance study. J Antimicrob Chemother. 2011;66(Suppl 3):iii69-80.

21. Sader HS, Flamm RK, Mendes RE, Farrell DJ, Jones RN. Antimicrobial activities of ceftaroline and comparator agents against bacterial organisms causing bacteremia in patients with skin and skin structure infections in U.S. Medical Centers, 2008 to 2014. Antimicrob Agents Chemother. 2016;60(4):2558-63.

22. Connor KA. Newer intravenous antibiotics in the intensive care unit: ceftaroline, ceftolozane-tazobactam, and ceftazidime-avibactam. AACN Adv Crit Care. 2016;27(4):353-7.

23. Kazmierczak KM, de Jonge BLM, Stone GG, Sahm DF. In vitro activity of ceftazidime/avibactam against isolates of Pseudomonas aeruginosa collected in European countries: INFORM global surveillance 2012-15. J Antimicrob Chemother. 2018;73(10):2777-81.

24. Sader HS, Flamm RK, Streit JM, Farrell DJ, Jones RN. Ceftaroline activity against bacterial pathogens frequently isolated in U.S. medical centers: results from five years of the AWARE surveillance program. Antimicrob Agents Chemother. 2015;59(4):2458-61.
25. Karlowsky JA, Biedenbach DJ, Bouchillon SK, Hackel M, laconis JP, Sahm DF. In vitro activity of Ceftaroline against bacterial pathogens isolated from patients with skin and soft tissue and respiratory tract infections in African and Middle Eastern countries: AWARE global surveillance program 2012-2014. Diagn Microbiol Infect Dis. 2016:86(2):194-9.

26. Bae IG, Stone GG. Activity of ceftaroline against pathogens associated with community-acquired pneumonia collected as part of the AWARE surveillance program, 2015-2016. Diagn Microbiol Infect Dis. 2019:95:114843.

27. Flamm RK, Sader HS, Farrell DJ, Jones RN. Ceftaroline potency among 9 US Census regions: report from the 2010 AWARE Program. Clin Infect Dis. 2012;55(Suppl 3):S194-205.

28. Mayor S. First WHO antimicrobial surveillance data reveal high levels of resistance globally. BMJ. 2018;360:k462.

29. World Health Organization. Global Antimicrobial Resistance Surveillance System (GLASS) report: early implementation 2016-17. 2017. https ://apps.who.int/iris/bitstream/10665/259744/1/9789241513449-eng. pdf?ua=1. Retrieved August 21, 2019. Geneva: World Health Organization; 2017.

30. World Health Organization. Antimicrobial resistance in the Asia Pacific region: a development agenda. Geneva: World Health Organization; 2017.

31. Kakkar M, Chatterjee P, Chauhan AS, et al. Antimicrobial resistance in South East Asia: time to ask the right questions. Glob Health Action. 2018;11(1):1483637

32. Chereau F, Opatowski L, Tourdjman M, Vong S. Risk assessment for antibiotic resistance in South East Asia. BMJ. 2017;358:j3393.

33. Jean SS, Hsueh PR. High burden of antimicrobial resistance in Asia. Int J Antimicrob Agents. 2011;37(4):291-5.

34. Kumarasamy KK, Toleman MA, Walsh TR, et al. Emergence of a new antibiotic resistance mechanism in India, Pakistan, and the UK: a molecular, biological, and epidemiological study. Lancet Infect Dis. 2010;10(9):597-602.

35. Zhang Z, Chen M, Yu Y, Pan S, Liu Y. Antimicrobial susceptibility among gram-positive and gram-negative blood-borne pathogens collected between 2012-2016 as part of the Tigecycline Evaluation and Surveillance Trial. Antimicrob Resist Infect Control. 2018;7:152.

36. O'Brien TF, Clark A, Peters R, Stelling J. Why surveillance of antimicrobial resistance needs to be automated and comprehensive. J Glob Antimicrob Resist. 2019;17:8-15.

37. Hendriksen RS, Munk P, Njage P, et al. Global monitoring of antimicrobial resistance based on metagenomics analyses of urban sewage. Nat Commun. 2019;10(1):1124

38. Laxminarayan $\mathrm{R}$, Duse A, Wattal C, et al. Antibiotic resistance-the need for global solutions. Lancet Infect Dis. 2013;13(12):1057-98.

39. Ranganathan P, Pramesh CS, Buyse M. Common pitfalls in statistical analysis: clinical versus statistical significance. Perspect Clin Res. 2015;6(3):169-70

40. Page P. Beyond statistical significance: clinical interpretation of rehabilitation research literature. Int J Sports Phys Ther. 2014;9(5):726-36.

\section{Publisher's Note}

Springer Nature remains neutral with regard to jurisdictional claims in published maps and institutional affiliations.

Ready to submit your research? Choose BMC and benefit from

- fast, convenient online submission

- thorough peer review by experienced researchers in your field

- rapid publication on acceptance

- support for research data, including large and complex data types

- gold Open Access which fosters wider collaboration and increased citations

- maximum visibility for your research: over 100M website views per year

At $\mathrm{BMC}$, research is always in progress.

Learn more biomedcentral.com/submissions 